\title{
Influencia de la edad, la maternidad y el empleo en las barreras para la práctica de actividad física y deporte de las mujeres adultas en España Influence of age, maternity and employment in barriers to practicing physical activity and sport for Spanish adult women
}

\author{
María Martín Rodríguez, María Isabel Barriopedro Moro, María Espada Mateos
}

Universidad Politécnica de Madrid (España)

\begin{abstract}
Resumen. Los objetivos del presente estudio fueron analizar la influencia de la edad y tipo de ocupación relacionada con el empleo y la crianza, en las barreras para la práctica de las mujeres adultas en España. La metodología de carácter cuantitativa, se ha apoyado en el uso de la encuesta mediante el empleo de un cuestionario basado en la teoría bio-ecológica de Bronfenbrenner, con ítems individuales, ocupación crianza-hogar, ocupación empleo-tiempo y oferta de actividad física y deporte, a una muestra representativa de 1.731 mujeres adultas españolas (entre 30 y 64 años). Los resultados evidenciaron efecto multivariado de la ocupación en las barreras ocupación-empleo y tiempo («No soy capaz de generar tiempo para mí», «empleo» $\mathrm{y}$ «horarios incompatibles»). Las mujeres adultas que concilian maternidad y empleo perciben en mayor grado estas tres barreras mencionadas. Además, se evidenció efecto multivariado de la edad y la ocupación, y de la interacción, en las barreras ocupación maternidad-hogar («Tengo que cuidar de mi/s hijo/as» y «Tengo que ocuparme de las tareas del hogar»). Las madres adultas, empleadas o no, percibieron la maternidad en mayor grado como barrera. En las empleadas que no son madres, las tareas del hogar como barrera tuvieron mayor prevalencia en las mayores de cincuenta años. Estas evidencias deberían considerarse para diseñar innovaciones y adaptar intervenciones, en el ámbito de la actividad física y el deporte, para estos grupos específicos, de mujeres adultas.
\end{abstract}

Palabras clave: Mujeres adultas, actividad física y deporte, barreras.

\begin{abstract}
The objectives of the present paper were to analyze the age and occupation type related to employment and maternity influencing Spanish adult women's barriers to practice. A quantitative methodology based on the use of the survey was applied to a representative sample of 1,731 Spanish adult women (between 30 and 64 years old) who filled out a questionnaire based on the Bronfenbrenner's bioecological theory with individual items, occupation maternity-household, occupation employment-time and physical activity and sport provision through a personal interview. The results showed a multivariate effect of the occupation on the occupation-employment and time barriers («I can't generate time for myself», «employment» and «incompatible timetables»). The adult women who combine maternity and employment perceived the three barriers mentioned to a greater extent. Also, there was found a multivariate effect of age and occupation, and of the interaction, on the occupation maternity-household barriers ("I have to take care of my children» and "I have to do the housework»). The adult mothers, employed or unemployed, perceived maternity as a barrier to a greater degree. In employed women who are not mothers, household chores as a barrier showed a higher prevalence in those over fifty years old. These findings should be considered for designing innovations and tailoring interventions in the field of physical activity and sport to fit these targeted groups of adult women.
\end{abstract}

Key words: Adult women, physical activity and sport, barriers.

\section{Introducción}

Las mujeres adultas en España continúan siendo uno de los grupos poblacionales que menos actividad física y deporte (en adelante AFD) practica (Ministerio de Cultura y Deporte, 2020; Moscoso \& Rodríguez, 2020). En una muestra representativa de la población española entre 30 y 64 años, Martín et al. (2014) indicaban que el 31\% de las mujeres practicaba ejercicio físico y de-

Fecha recepción: 28-02-21. Fecha de aceptación: 11-12-21

María Martín Rodríguez

maria.martin@upm.es porte, un 26\% principalmente se ejercitaban caminando y que un $24 \%$ aunque no practicaba, deseaba hacerlo, siendo mayoritariamente madres empleadas. En este sentido, la actividad física se presenta relacionada con la percepción de barreras o factores que obstaculizan o impiden la práctica deportiva (Espada y Galán, 2017). McGuire, Seib y Anderson (2016), evidenciaron también que en mujeres adultas la percepción de barreras influye en su práctica de AFD.

La confluencia del empleo con las tareas de cuidado de familia y hogar, tradicionalmente atribuidas a las mujeres, limitan de manera importante su disponibilidad de tiempo libre para la práctica de AFD. Moscoso y 
Rodríguez (2020) señalaban que esta triple jornada o rol femenino contribuye a explicar la «clara persistencia de desigualdades sociales crónicas (o "brecha deportiva») en relación al género y la edad» (Moscoso \& Rodríguez, 2020, pp. 199). Este triple rol o jornada femenina parece también explicar que la falta de tiempo es la principal barrera para la práctica de AFD en la Unión Europea (40\%) siendo superior en la población española (47\%) y alcanzando sus valores más elevados en las mujeres entre 25 y 39 años (57\%) (European Commission, 2018).

Diversas investigaciones han corroborado que la falta de tiempo aparece entre las principales barreras para la práctica deAFD de mujeres y hombres adultos (Amin, Suleman, Ali, Gamal \& Al Wehedy, 2011; Bautista, Reininger, Gay, Barroso \& McCormick, 2011; Cerin, Leslie, Sugiyama \& Owen, 2010; De Bourdeaudhuij \& Sallis, 2002; Louw, Van Biljon, \& Mugandani, 2012; Sebastião, Pinto, Santos, Marques \& Carreiro da Costa, 2011; Seefeldt, Malina \& Clark, 2002; Sørensen \& Gill, 2008). Desde una perspectiva ecológica, algunos de estos estudios previos señalaron la influencia de los tradicionales roles de género en las barreras, ya que las mujeres presentaban en mayor medida que los hombres la barrera cuidado de sus hijos y hogar, mientras que ellos indicaban en mayor medida que ellas, la incompatibilidad de horarios con el empleo (Amin et al., 2011; Cerin et al., 2010; Sorensen \& Gill, 2008).

En España, recientes investigaciones confirman que las mujeres en general siguen disponiendo de menos tiempo libre que los hombres porque ellas siguen asumiendo la mayor parte de las responsabilidades de cuidado y tareas del hogar, aunque se haya ampliado su participación en el mercado laboral (Instituto de la Mujer y para la Igualdad de oportunidades, 2020b). En lo que respecta a sus hábitos deportivos, las mujeres adultas son menos propensas a hacer deporte los días laborales, si bien, con una edad avanzada tanto hombres como mujeres tienden a hacer menos a AFD durante el fin de semana, lo que se debe posiblemente a la mayor cantidad de tiempo libre que los adultos disponen durante los días laborables para actividades de ocio a medida que envejecen (Espada et al., 2018).

En mujeres adultas españolas, varios estudios indicaban que las barreras principales para la práctica de AFD eran la falta de tiempo, las tareas del hogar y de cuidado de hijos/as o crianza y el empleo como barreras para la práctica de AFD (Martínez del Castillo et al., 2005; Moscoso et al., 2008), siendo la crianza más importante para las adultas más jóvenes (Moscoso et al., 2008). Este hecho pudiera explicarse por la mayor exigencia de cui- dados requeridos en los primeros años de vida de su/s hija/os, los drásticos cambios en las condiciones de vida que conlleva la maternidad, unido a los déficits de corresponsabilidad y conciliación que todavía presentan parte de los hombres y la sociedad española en su conjunto, incluidos los sistemas laboral, educativo y deportivo. En relación a esta cuestión, García Ferrando y Llopis (2011) señalaban que uno de los dos importantes momentos de abandono de la práctica de las mujeres se producía por la confluencia de maternidad y empleo. En mujeres adultas latinas, estudios cualitativos señalan que la persistencia de roles de género como la ética del cuidado, o anteponer el cuidado de la familia al cuidado propio, ha sido identificada como una de sus barreras más importantes para la práctica de AFD (Gonzales, 2004).

Actualmente, a estas dificultades habituales de las mujeres adultas para conciliar práctica de AFD con empleo y crianza, hay que añadir las consecuencias derivadas de la pandemia por el COVID-19. También en España, su impacto ha sido mayor en las mujeres adultas por el incremento de las tareas de cuidado familiar y doméstico, la sobrecarga del feminizado empleo sanitario y de servicios esenciales, su mayor precariedad laboral, pobreza, así como el aumento del riesgo de sufrir violencia de género (Instituto de la Mujer y para la Igualdad de oportunidades, 2020a). Durante el confinamiento domiciliario las tareas de cuidado se han centralizado en los hogares y en las mujeres, al limitarse las redes de apoyo comunitarias y familiares. Parte de las madres empleadas han tenido que conciliar teletrabajo y telecolegio, otras no han podido seguir trabajando. Por la fatiga, este impacto se amplía a medida que aumenta la duración de esta pandemia COVID-19. CastañedaBarbarro, Arbillaga-Etxarri, Gutiérrez-Santamaría y Coca (2020) y García, Sahelices, Mendaña y Magaz (2020) atribuyeron a esta sobrecarga por déficits de corresponsabilidad en las tareas de cuidado los diferentes cambios en los comportamientos de práctica de AFD de mujeres y hombres adultos españoles durante el confinamiento domiciliario.

En relación a otros cambios vinculados al COVID19, algunas organizaciones lanzaron su oferta de AFD en entornos web, con lo que ciertas actividades habituales se pudieron mantener: entrenamiento, readaptación funcional, salud, recreación, etc. (García-Tascón, Mendaña-Cuervo, Sahelices-Pinto \& Magaz-González, 2021). Por su parte, García et al. (2020) señaló que la práctica virtual fue significativamente mayor entre las mujeres. Piedra (2020) indicaba que las influencers del ejercicio físico online principalmente seguidas por mu- 
jeres, como Patry Jordan en España, aunque la mayoría carece de titulación profesional para dirigir práctica de AFD, partían mejor posicionadas e incrementaron en mayor medida que los influencers su seguimiento. En este periodo se permitió salir a los niños y niñas a pasear acompañados de una/a progenitor/a. Se presentaron aglomeraciones y riesgo de accidentes pero también novedosas acciones adaptativas que señalan la necesidad y oportunidad de una transformación urbana facilitadora del ejercicio físico y el movimiento lúdico al aire libre, de práctica conjunta o coordinada adultas-infancia, en espacios más saludables, orgánicos y seguros (Sánchez García, 2020).

Organizaciones de referencia, como el Grupo internacional Mujeres y deporte, mediante su $I W G$ responds to COVID 19 with global 'Call to Action'recomiendan, especialmente a las organizaciones firmantes de la Brighton plus Helsinki 2014 Declaration on Women \& Sport, promover el regreso a la AFD fuera de casa tan pronto como sea segura. Mientras tanto, priorizan moverse en el hogar, así como recrear actividades y espacios seguros para las mujeres, contando con su participación para reimaginar y organizar cómo afrontar este reto con seguridad y equidad.

Finalmente, a pesar de que se han realizado investigaciones desde la perspectiva ecológica y perspectiva de género (Abbasi, 2014, Cerin et al., 2010; Seefeldt, Malina \& Clark, 2002), en los que se tiene presente los modelos ecológicos los cuales presentan una visión amplia de la causalidad del comportamiento de salud, con el entorno social y físico incluidos como contribuyentes a la inactividad física (Bauman et al., 2012), no se han encontrado investigaciones que analicen la influencia de determinantes individuales, familiares, sociales y culturales, como la crianza y el empleo, que afectan de manera diferente a las mujeres adultas.

Por ello, los objetivos del presente estudio fueron analizar la influencia de la edad y tipo de ocupación relacionada con la maternidad o crianza y el empleo, en las barreras para la práctica de AFD de las mujeres adultas en España.

\section{Material y método}

El planteamiento metodológico de la presente investigación fue de carácter cuantitativo. La técnica de recogida de información consistió en el uso de entrevistas estructuradas a una muestra estadísticamente representativa de las mujeres y hombres entre 30 y 64 años en España (47.265.321 personas adultas, 23.966.965 mujeres y 23.298.356 hombres, según el Instituto Nacional de Estadística, Padrón Municipal 2012). Dado que es una población infinita o muy numerosa, y trabajando con un intervalo de confianza del 95.5\%, y suponiendo en la varianza poblacional el caso más desfavorable de $p$ igual a $q$, el margen de error de muestreo fue de \pm 1.7\%. El tipo de muestreo fue probabilístico, de tipo polietápico, siendo las unidades de primera etapa los municipios en que residían habitualmente las personas adultas. La afijación de la muestra fue proporcional a la distribución de las personas adultas según tamaño demográfico de los municipios y según género. El trabajo de campo se llevó a cabo durante los meses de octubre a diciembre de 2012, enero y febrero 2013 y de octubre a diciembre de 2013. En él, las y los entrevistadores aplicaron el cuestionario escrito mediante entrevista personal estructurada cara a cara, en el domicilio habitual de la persona adulta.

La muestra total registra las siguientes características: el tamaño de la muestra final ha sido de 3.463 personas adultas, 1.732 hombres (edad media 46.6 años \pm 9,7) y 1.731 mujeres (edad media 44.5 años $\pm 9,6$ ).

Para el presente trabajo, la muestra fue de 1.731 mujeres con edades comprendidas entre los 30 y los 64 años cumplidos $(45,32 \pm 9,7)$ y residentes en España

Se recogieron las variables: edad, ocupación empleo, ocupación crianza de hijo/as. Las barreras se recogieron mediante un cuestionario específico sobre barreras para la práctica de actividad física en personas adultas de 11 ítems, agrupados en 4 factores mediante análisis factorial (Ver Tabla 1) de acuerdo a la Teoría ecológica de Bronfenbrenner (1993). Esta teoría postula que el comportamiento y desarrollo individual están determinados por un proceso multi-sistémico de la persona que actúa desde diferentes niveles (cultural, social, familiar e individual) los cuales constantemente interactúan y se influencian mutuamente. Tras los respectivos análisis factoriales, exploratorios y confirmatorios el análisis factorial agrupó lo ítems en cuatro dimensiones diferentes, apareciendo dos de ellas relacionadas: Dimensión 1 Individuales, Dimensión 2 Ocupación crianza y hogar, Dimensión 3 Ocupación Empleo y tiempo y Dimensión 4 Oferta de AFD. Todas ellas presentaron un índice de fiabilidad a través del alfa de Cronbach superior al .7. Los resultados muestran un buen ajuste al modelo presentado $(\mathrm{RMSEA}=.047$ (.038-.056); $\left.\mathrm{CFI}=.96 ; \div_{(42)}^{2}=133.1\right)$. Las dimensiones relacionadas fueron la 2 y 3 , vinculadas ambas a ocupaciones y diferenciadas por agrupar la 2 las tareas de cuidado de familia y hogar, tradicionales roles de cuidado atribuidos a 
las mujeres.

Los datos fueron analizados mediante el programa estadístico SPSS versión 18, estableciéndose grupos en función de la edad (35 años o menos, 36 a 49 años y más de 50 años) y tipo de ocupación (SÍ empleo-SÍ crianza, SÍ empleo-NO crianza, NO empleo-SÍ crianza, NO empleo-NO crianza). Para analizar la presencia de las distintas barreras consideradas en función de la edad y el tipo de ocupación se realizaron diferentes análisis de estadística descriptiva e inferencial. Se utilizaron MANOVAS de 2 factores intersujetos. Las comparaciones múltiples a posteriori se realizaron mediante la prueba de Bonferroni. Como índice del tamaño del efecto se calculó eta cuadrado parcial (ç2 2 ). La interpretación de ç2 2 se basó en los siguientes valores: $<0,06$ pequeño, e» 0,06 hasta $<0,14$ medio y e» 0,14 grande. El nivel de significación se fijó en .05 .

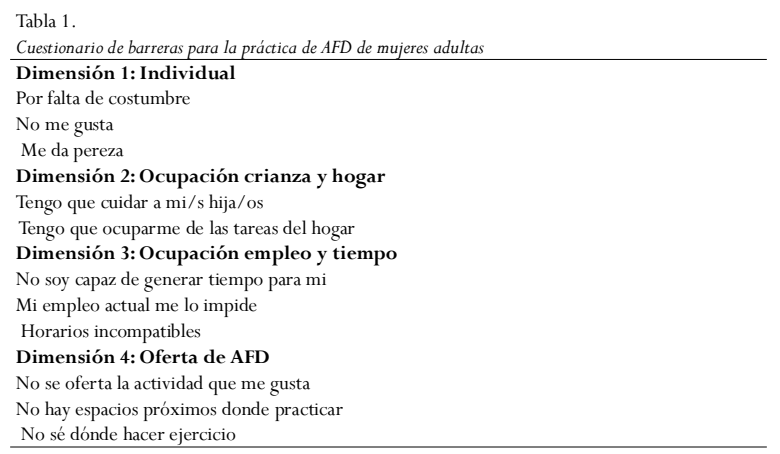

\section{Resultados}

\section{Barreras de la Dimensión 1: Individual}

No hubo efecto multivariado de la edad $\left(\mathrm{F}_{6,2068}=1.86 ; p=.085\right)$, ni de la ocupación $\left(\mathrm{F}_{9,3105}=1.62\right.$; $p=.105)$, ni de la interacción $\left(\mathrm{F}_{18,3105}=01.06 ; p=.384\right)$, sobre las barreras individuales o personales.

\section{Barreras de la Dimensión 2: Ocupación crianza} y hogar

Hubo efecto multivariado de la edad $\left(\mathrm{F}_{4,2084}=11.19\right.$; $p<.001)$. Los contrastes univariados pusieron de manifiesto diferencias en función de la edad para la barrera «Tengo que cuidar de mi /s hija / os» $\left(\mathrm{F}_{2,1042}=16.46 ; p<.001\right.$; $\left.\chi_{\mathrm{p}}^{2}=0,03\right)$, pero no para la barrera «Tengo que ocuparme de las tareas del hogar» $\left(\mathrm{F}_{2,1042}=0.018 ; p=.983\right)$. Las comparaciones múltiples pusieron de manifiesto que los grupos de mujeres más jóvenes (35 o menos) presentaron en mayor medida la crianza como barrera para la práctica de actividad física y/o deporte que el grupo de mujeres de 36 a 49 y de 50 años o más $(p<.05$ para ambas comparaciones) algo que estará en consonancia
Tabla 2.

\begin{tabular}{|c|c|c|c|c|c|}
\hline Barrera & Grupo edad & Ocupación & Media & Dt & $\mathrm{N}$ \\
\hline \multirow{20}{*}{ Falta de costumbre } & \multirow{5}{*}{35 o menos } & SI empleo-SI crianza & .87 & 1.097 & 78 \\
\hline & & SI empleo-NO crianza & 1.36 & 1.262 & 76 \\
\hline & & NO empleo-SI crianza & 1.16 & 1.242 & 38 \\
\hline & & NO empleo-NO crianza & 1.36 & 1.168 & 33 \\
\hline & & Total & 1.16 & 1.202 & 225 \\
\hline & \multirow{5}{*}{36 a 49} & SI empleo-SI crianza & .97 & 1.133 & 265 \\
\hline & & SI empleo-NO crianza & 1.08 & 1.175 & 102 \\
\hline & & NO empleo-SI crianza & 1.05 & 1.196 & 105 \\
\hline & & NO empleo-NO crianza & 1.26 & 1.251 & 42 \\
\hline & & Total & 1.03 & 1.164 & 514 \\
\hline & \multirow{5}{*}{50 o más } & SI empleo-SI crianza & 1.00 & 1.247 & 37 \\
\hline & & SI empleo-NO crianza & 1.05 & 1.193 & 107 \\
\hline & & NO empleo-SI crianza & 1.02 & 1.193 & 41 \\
\hline & & NO empleo-NO crianza & 1.15 & 1.235 & 123 \\
\hline & & Total & 1.08 & 1.212 & 308 \\
\hline & \multirow{5}{*}{ Total } & SI empleo-SI crianza & .95 & 1.135 & 380 \\
\hline & & SI empleo-NO crianza & 1.14 & 1.208 & 285 \\
\hline & & NO empleo-SI crianza & 1.07 & 1.199 & 184 \\
\hline & & NO empleo-NO crianza & 1.21 & 1.224 & 198 \\
\hline & & Total & 1.07 & 1.186 & 1047 \\
\hline \multirow{20}{*}{$\begin{array}{l}\text { No me gusta hacer ejercicio } \\
\text { deporte }\end{array}$} & \multirow{5}{*}{35 o menos } & SI empleo-SI crianza & .40 & .811 & 78 \\
\hline & & SI empleo-NO crianza & .58 & .913 & 76 \\
\hline & & NO empleo-SI crianza & .76 & 1.125 & 38 \\
\hline & & NO empleo-NO crianza & .39 & .899 & 33 \\
\hline & & Total & .52 & .921 & 225 \\
\hline & \multirow{5}{*}{36 a 49} & SI empleo-SI crianza & .55 & .976 & 265 \\
\hline & & SI empleo-NO crianza & .65 & 1.011 & 102 \\
\hline & & NO empleo-SI crianza & .82 & 1.175 & 105 \\
\hline & & NO empleo-NO crianza & .71 & .970 & 42 \\
\hline & & Total & .64 & 1.029 & 514 \\
\hline & \multirow{5}{*}{50 o más } & SI empleo-SI crianza & .97 & 1.258 & 37 \\
\hline & & SI empleo-NO crianza & .64 & 1.057 & 107 \\
\hline & & NO empleo-SI crianza & .59 & 1.024 & 41 \\
\hline & & NO empleo-NO crianza & .76 & 1.133 & 123 \\
\hline & & Total & .72 & 1.110 & 308 \\
\hline & \multirow{5}{*}{ Total } & SI empleo-SI crianza & .56 & .985 & 380 \\
\hline & & SI empleo-NO crianza & .63 & 1.001 & 285 \\
\hline & & NO empleo-SI crianza & .76 & 1.131 & 184 \\
\hline & & NO empleo-NO crianza & .69 & 1.068 & 198 \\
\hline & & Total & .64 & 1.033 & 1047 \\
\hline \multirow{20}{*}{ Me da pereza } & \multirow{5}{*}{35 o menos } & SI empleo-SI crianza & .83 & 1.086 & 78 \\
\hline & & SI empleo-NO crianza & 1.14 & 1.186 & 76 \\
\hline & & NO empleo-SI crianza & 1.39 & 1.326 & 38 \\
\hline & & NO empleo-NO crianza & 1.12 & 1.193 & 33 \\
\hline & & Total & 1.08 & 1.187 & 225 \\
\hline & \multirow{5}{*}{36 a 49} & SI empleo-SI crianza & 1.12 & 1.185 & 265 \\
\hline & & SI empleo-NO crianza & 1.18 & 1.262 & 102 \\
\hline & & NO empleo-SI crianza & 1.18 & 1.329 & 105 \\
\hline & & NO empleo-NO crianza & 1.64 & 1.358 & 42 \\
\hline & & Total & 1.19 & 1.249 & 514 \\
\hline & \multirow{5}{*}{50 o más } & SI empleo-SI crianza & 1.11 & 1.149 & 37 \\
\hline & & SI empleo-NO crianza & 1,17 & 1.270 & 107 \\
\hline & & NO empleo-SI crianza & 1.24 & 1.300 & 41 \\
\hline & & NO empleo-NO crianza & 1.32 & 1.301 & 123 \\
\hline & & Total & 1.23 & 1.269 & 308 \\
\hline & \multirow{5}{*}{ Total } & SI empleo-SI crianza & 1.06 & 1.165 & 380 \\
\hline & & SI empleo-NO crianza & 1.16 & 1.241 & 285 \\
\hline & & NO empleo-SI crianza & 1.24 & 1.317 & 184 \\
\hline & & NO empleo-NO crianza & 1.35 & 1.301 & 198 \\
\hline & & Total & 1.18 & 1.242 & 1047 \\
\hline
\end{tabular}

con la edad media de maternidad para el/la primer/a hijo/a en España.

Hubo efecto multivariado del tipo de ocupación $\left(\mathrm{F}_{6,2084}=70.13 ; p<.001\right)$. Los contrastes univariados pusieron de manifiesto diferencias en función de la ocupación para la barrera «Tengo que cuidar de $\mathrm{mi} / \mathrm{s}$ hija/os» $\left(\mathrm{F}_{3,1042}=164.45 ; p<.001 ; \chi_{\mathrm{p}}^{2}=0,32\right)$ y para la barrera «Tengo que ocuparme de las tareas del hogar» $\left(\mathrm{F}_{3,1042}=23.19\right.$; $\left.p<.001 ; \chi_{\mathrm{p}}^{2}=0,06\right)$. Las comparaciones múltiples pusieron de manifiesto que los grupos de mujeres que trabajaban y tenían hijos (doble jornada) puntuaron en mayor medida ambas barreras en comparación con aquellas mujeres que solo tenían empleo o eran madres no empleadas $(p<.05$ para todas las comparaciones).

Por último, hubo efecto multivariado de la interacción entre la edad y el tipo de ocupación $\left(\mathrm{F}_{12,2084}=4.53\right.$; 
$p<.001)$. Los análisis univariados mostraron efecto significativo de la interacción tanto para la barrera «Tengo que cuidar de mi/s hija/os» $\left(\mathrm{F}_{6,1042}=8.87 ; p<.001 ; \chi_{\mathrm{p}}^{2}=\right.$ 0,05 ) como para la barrera «Tengo que ocuparme de las tareas del hogar» $\left(\mathrm{F}_{6,1042}=3.79 ; p<.001 ; \chi_{\mathrm{p}}^{2}=0,02\right)$. Tanto para las madres que concilian empleo y crianza como para aquellas no empleadas con crianza, se observa que la barrera relacionada con el cuidado de sus hija/os se presenta en mayor medida cuanto menor es la edad de las mujeres. En relación a la barrera relacionada con las tareas del hogar, solo se observan diferencias en función de la edad en el grupo de mujeres empleadas que no son madres. Esta barrera se presenta en mayor medida en las mujeres más mayores (50 años y más).

Tabla 3.

\begin{tabular}{|c|c|c|c|c|c|}
\hline Barrera & Grupo edad & Ocupación & Media & Dt & $\mathrm{N}$ \\
\hline \multirow{20}{*}{$\begin{array}{l}\text { Tengo que cuidar de } \\
\mathrm{mi} / \mathrm{s} \mathrm{hija} / \mathrm{os}\end{array}$} & \multirow{5}{*}{35 o menos } & SI empleo-SI crianza & 2.27 & 1.136 & 78 \\
\hline & & SI empleo-NO crianza & .09 & .401 & 78 \\
\hline & & NO empleo-SI crianza & 2.29 & 1.160 & 38 \\
\hline & & NO empleo-NO crianza & .06 & .242 & 33 \\
\hline & & Total & 1.20 & 1.390 & 227 \\
\hline & \multirow{5}{*}{36 a 49} & SI empleo-SI crianza & 1.85 & 1.217 & 265 \\
\hline & & SI empleo-NO crianza & .19 & .611 & 103 \\
\hline & & NO empleo-SI crianza & 1.74 & 1.312 & 106 \\
\hline & & NO empleo-NO crianza & .07 & .258 & 43 \\
\hline & & Total & 1.35 & 1.323 & 517 \\
\hline & \multirow{5}{*}{50 o más } & SI empleo-SI crianza & 1.16 & 1.344 & 37 \\
\hline & & SI empleo-NO crianza & .26 & .805 & 107 \\
\hline & & NO empleo-SI crianza & .98 & 1.259 & 42 \\
\hline & & NO empleo-NO crianza & .15 & .585 & 124 \\
\hline & & Total & .42 & .961 & 310 \\
\hline & \multirow{5}{*}{ Total } & SI empleo-SI crianza & 1.87 & 1.244 & 380 \\
\hline & & SI empleo-NO crianza & .19 & .648 & 288 \\
\hline & & NO empleo-SI crianza & 1.68 & 1.337 & 186 \\
\hline & & NO empleo-NO crianza & .12 & .487 & 200 \\
\hline & & Total & 1.05 & 1.307 & 1054 \\
\hline \multirow{20}{*}{$\begin{array}{l}\text { Tengo que ocuparme } \\
\text { de las tareas del hogar }\end{array}$} & \multirow{5}{*}{35 o menos } & SI empleo-SI crianza & 1.88 & 1.044 & 78 \\
\hline & & SI empleo-NO crianza & .83 & 1.110 & 78 \\
\hline & & NO empleo-SI crianza & 1.87 & 1.319 & 38 \\
\hline & & NO empleo-NO crianza & .58 & .936 & 33 \\
\hline & & Total & 1.33 & 1.234 & 227 \\
\hline & \multirow{5}{*}{36 a 49} & SI empleo-SI crianza & 1.65 & 1.159 & 265 \\
\hline & & SI empleo-NO crianza & 1.09 & 1.121 & 103 \\
\hline & & NO empleo-SI crianza & 1.65 & 1.324 & 106 \\
\hline & & NO empleo-NO crianza & .70 & .832 & 43 \\
\hline & & Total & 1.46 & 1.204 & 517 \\
\hline & \multirow{5}{*}{50 o más } & SI empleo-SI crianza & 1.46 & 1.216 & 37 \\
\hline & & SI empleo-NO crianza & 1.27 & 1.154 & 107 \\
\hline & & NO empleo-SI crianza & 1.26 & 1.289 & 42 \\
\hline & & NO empleo-NO crianza & 1.11 & 1.142 & 124 \\
\hline & & Total & 1.23 & 1.175 & 310 \\
\hline & \multirow{5}{*}{ Total } & SI empleo-SI crianza & 1.68 & 1.145 & 380 \\
\hline & & SI empleo-NO crianza & 1.09 & 1.140 & 288 \\
\hline & & NO empleo-SI crianza & 1.61 & 1.324 & 186 \\
\hline & & NO empleo-NO crianza & .94 & 1.071 & 200 \\
\hline & & Total & 1.36 & 1.205 & 1054 \\
\hline
\end{tabular}

\section{Barreras de la Dimensión 3: Ocupación empleo y tiempo}

Los resultados no muestran la existencia de efecto multivariado de la edad $\left(\mathrm{F}_{6,2080}=61.30 ; p<.253\right)$ pero sí de la ocupación $\left(\mathrm{F}_{9,3123}=22.83 ; p<.001\right)$. No hubo efecto de la interacción $\left(\mathrm{F}_{18,3123}=1.55 ; p<.066\right)$.

Los contrastes univariados pusieron de manifiesto diferencias en función de la ocupación para la barrera «No saco tiempo para mi» $\left(\mathrm{F}_{3.1041}=26.56 ; p<.001\right)$. Las madres empleadas presentan en mayor medida esta barrera que los otros tres grupos, y las empleadas que no son madres o están desempleadas también la presentan en mayor medida que las desempleadas que no son madres ( $p<.05$ para todas las comparaciones).

Los contrastes univariados pusieron de manifiesto diferencias en función de la ocupación para la barrera «Mi empleo no me lo permite» $\left(\mathrm{F}_{3.1041}=56.99 ; p<.001 ; \chi_{\mathrm{p}}^{2}\right.$ $=0,15)$. Las comparaciones múltiples pusieron de manifiesto que los grupos de mujeres empleadas, con o sin crianza, presentan en mayor medida esta barrera que los otros dos grupos $(p<.05$ para todas las comparaciones).

Los contrastes univariados pusieron de manifiesto diferencias en función de la ocupación para la barrera «Horarios incompatibles» $\left(\mathrm{F}_{3.1041}=19.32 ; p<.001 ; \chi_{\mathrm{p}}^{2}=\right.$ $0,01)$. Los grupos de mujeres empleadas, con o sin crianza presentan en mayor medida esta barrera que los otros dos grupos ( $p<.05$ para todas las comparaciones).

\begin{tabular}{|c|c|c|c|c|c|}
\hline Barrera & Grupo edad & Ocupación & Media & DT & $\mathrm{N}$ \\
\hline \multirow{20}{*}{$\begin{array}{l}\text { No soy capaz de } \\
\text { generar tiempo para mi }\end{array}$} & \multirow{5}{*}{35 o menos } & SI empleo-SI crianza & 2.23 & 1.031 & 78 \\
\hline & & SI empleo-NO crianza & 1.92 & 1.090 & 78 \\
\hline & & NO empleo-SI crianza & 1.87 & 1.234 & 38 \\
\hline & & NO empleo-NO crianza & .97 & 1.237 & 33 \\
\hline & & Total & 1.88 & 1.182 & 227 \\
\hline & \multirow{5}{*}{36 a 49} & SI empleo-SI crianza & 2.38 & 1.020 & 264 \\
\hline & & SI empleo-NO crianza & 1.74 & 1.260 & 103 \\
\hline & & NO empleo-SI crianza & 1.79 & 1.300 & 106 \\
\hline & & NO empleo-NO crianza & 1.02 & 1.102 & 43 \\
\hline & & Total & 2.02 & 1.210 & 516 \\
\hline & \multirow{5}{*}{50 o más } & SI empleo-SI crianza & 2.05 & 1.201 & 37 \\
\hline & & SI empleo-NO crianza & 1.91 & 1.217 & 107 \\
\hline & & NO empleo-SI crianza & 1.57 & 1.272 & 42 \\
\hline & & NO empleo-NO crianza & 1.29 & 1.348 & 124 \\
\hline & & Total & 1.63 & 1.307 & 310 \\
\hline & \multirow{5}{*}{ Total } & SI empleo-SI crianza & 2.31 & 1.044 & 379 \\
\hline & & SI empleo-NO crianza & 1.85 & 1.199 & 288 \\
\hline & & NO empleo-SI crianza & 1.76 & 1.278 & 186 \\
\hline & & NO empleo-NO crianza & 1.18 & 1.283 & 200 \\
\hline & & Total & 1.87 & 1.243 & 1053 \\
\hline \multirow{20}{*}{$\begin{array}{l}\text { Mi empleo actual me lo } \\
\text { impide }\end{array}$} & \multirow{5}{*}{35 o menos } & SI empleo-SI crianza & 1.44 & 1.265 & 78 \\
\hline & & SI empleo-NO crianza & 1.71 & 1.239 & 78 \\
\hline & & NO empleo-SI crianza & .32 & .809 & 38 \\
\hline & & NO empleo-NO crianza & .70 & 1.075 & 33 \\
\hline & & Total & 1.23 & 1.270 & 227 \\
\hline & \multirow{5}{*}{36 a 49} & SI empleo-SI crianza & 1.53 & 1.227 & 264 \\
\hline & & SI empleo-NO crianza & 1.19 & 1.276 & 103 \\
\hline & & NO empleo-SI crianza & .34 & .791 & 106 \\
\hline & & NO empleo-NO crianza & .26 & .658 & 43 \\
\hline & & Total & 1.11 & 1.237 & 516 \\
\hline & \multirow{5}{*}{50 o más } & SI empleo-SI crianza & 1.30 & 1.244 & 37 \\
\hline & & SI empleo-NO crianza & 1.37 & 1.321 & 107 \\
\hline & & NO empleo-SI crianza & .33 & .874 & 42 \\
\hline & & NO empleo-NO crianza & .20 & .698 & 124 \\
\hline & & Total & .75 & 1.179 & 310 \\
\hline & \multirow{5}{*}{ Total } & SI empleo-SI crianza & 1.49 & 1.235 & 379 \\
\hline & & SI empleo-NO crianza & 1.40 & 1.295 & 288 \\
\hline & & NO empleo-SI crianza & .33 & .810 & 186 \\
\hline & & NO empleo-NO crianza & .30 & .782 & 200 \\
\hline & & Total & 1.03 & 1.240 & 1053 \\
\hline \multirow{20}{*}{ Horarios incompatibles } & \multirow{5}{*}{35 o menos } & SI empleo-SI crianza & 1.51 & 1.203 & 78 \\
\hline & & SI empleo-NO crianza & 1.46 & 1.213 & 78 \\
\hline & & NO empleo-SI crianza & .89 & 1.110 & 38 \\
\hline & & NO empleo-NO crianza & 1.00 & 1.199 & 33 \\
\hline & & Total & 1.32 & 1.211 & 227 \\
\hline & \multirow{5}{*}{36 a 49} & SI empleo-SI crianza & 1.67 & 1.213 & 264 \\
\hline & & SI empleo-NO crianza & 1.09 & 1.292 & 103 \\
\hline & & NO empleo-SI crianza & 93 & 1.205 & 106 \\
\hline & & NO empleo-NO crianza & .37 & .725 & 43 \\
\hline & & Total & 1.29 & 1.264 & 516 \\
\hline & \multirow{5}{*}{50 o más } & SI empleo-SI crianza & 1.30 & 1.309 & 37 \\
\hline & & SI empleo-NO crianza & 1.35 & 1.332 & 107 \\
\hline & & NO empleo-SI crianza & .60 & 1.037 & 42 \\
\hline & & NO empleo-NO crianza & .63 & 1.032 & 124 \\
\hline & & Total & .95 & 1.226 & 310 \\
\hline & \multirow{5}{*}{ Total } & SI empleo-SI crianza & 1.60 & 1.223 & 379 \\
\hline & & SI empleo-NO crianza & 1.28 & 1.291 & 288 \\
\hline & & NO empleo-SI crianza & .85 & 1.153 & 186 \\
\hline & & NO empleo-NO crianza & .64 & 1.018 & 200 \\
\hline & & Total & 1.20 & 1.250 & 1053 \\
\hline
\end{tabular}




\section{Barreras de la Dimensión 4: Oferta de AFD}

No hubo efecto multivariado de la edad $\left(\mathrm{F}_{6,2082}=1.62\right.$; $p=.139)$, ni de la ocupación $\left(\mathrm{F}_{9,3126}=1.63 ; p=.101\right), \mathrm{ni}$ de la interacción $\left(\mathrm{F}_{18,3126}=0.57 ; p=.923\right)$, sobre las barreras relacionadas con la oferta de AFD.

\begin{tabular}{|c|c|c|c|c|c|}
\hline Barrera & Grupo edad & Ocupación & Media & $\mathrm{Dt}$ & $\mathrm{N}$ \\
\hline \multirow{20}{*}{$\begin{array}{l}\text { No se oferta actividad } \\
\text { que me gusta }\end{array}$} & \multirow{5}{*}{35 o menos } & SI empleo-SI crianza & .44 & .877 & 78 \\
\hline & & SI empleo-NO crianza & .46 & .935 & 78 \\
\hline & & NO empleo-SI crianza & .63 & 1,125 & 38 \\
\hline & & NO empleo-NO crianza & .55 & 1.034 & 33 \\
\hline & & Total & .49 & .961 & 227 \\
\hline & \multirow{5}{*}{36 a 49} & SI empleo-SI crianza & .43 & .935 & 265 \\
\hline & & SI empleo-NO crianza & .59 & 1,052 & 103 \\
\hline & & NO empleo-SI crianza & .62 & 1.099 & 106 \\
\hline & & NO empleo-NO crianza & .67 & 1.040 & 43 \\
\hline & & Total & .52 & 1.005 & 517 \\
\hline & \multirow{5}{*}{50 o más } & SI empleo-SI crianza & .32 & .784 & 37 \\
\hline & & SI empleo-NO crianza & .31 & .794 & 107 \\
\hline & & NO empleo-SI crianza & .38 & .936 & 42 \\
\hline & & NO empleo-NO crianza & .34 & .825 & 124 \\
\hline & & Total & .33 & .822 & 310 \\
\hline & \multirow{5}{*}{ Total } & SI empleo-SI crianza & .42 & .908 & 380 \\
\hline & & SI empleo-NO crianza & .45 & .936 & 288 \\
\hline & & NO empleo-SI crianza & .57 & 1.069 & 186 \\
\hline & & NO empleo-NO crianza & .45 & .917 & 200 \\
\hline & & Total & .46 & .948 & 1054 \\
\hline \multirow{20}{*}{$\begin{array}{l}\text { No se donde hacer } \\
\text { ejercicio }\end{array}$} & \multirow{5}{*}{35 o menos } & SI empleo-SI crianza & .54 & .963 & 78 \\
\hline & & SI empleo-NO crianza & .65 & 1.017 & 78 \\
\hline & & NO empleo-SI crianza & .58 & 1.081 & 38 \\
\hline & & NO empleo-NO crianza & .64 & 1.113 & 33 \\
\hline & & Total & .60 & 1.019 & 227 \\
\hline & \multirow{5}{*}{36 a 49} & SI empleo-SI crianza & .48 & .946 & 265 \\
\hline & & SI empleo-NO crianza & .51 & .969 & 103 \\
\hline & & NO empleo-SI crianza & .82 & 1.241 & 106 \\
\hline & & NO empleo-NO crianza & .72 & 1.120 & 43 \\
\hline & & Total & .57 & 1.038 & 517 \\
\hline & \multirow{5}{*}{50 o más } & SI empleo-SI crianza & .41 & .896 & 37 \\
\hline & & SI empleo-NO crianza & .38 & .938 & 107 \\
\hline & & NO empleo-SI crianza & .60 & 1.127 & 42 \\
\hline & & NO empleo-NO crianza & .54 & 1.007 & 124 \\
\hline & & Total & .48 & .988 & 310 \\
\hline & \multirow{5}{*}{ Total } & SI empleo-SI crianza & .48 & .943 & 380 \\
\hline & & SI empleo-NO crianza & .50 & .974 & 288 \\
\hline & & NO empleo-SI crianza & .72 & 1.184 & 186 \\
\hline & & NO empleo-NO crianza & .60 & 1.047 & 200 \\
\hline & & Total & .55 & 1.020 & 1054 \\
\hline \multirow{20}{*}{$\begin{array}{l}\text { No tengo espacios } \\
\text { próximos para } \\
\text { practicar }\end{array}$} & \multirow{5}{*}{35 o menos } & SI empleo-SI crianza & .31 & .726 & 78 \\
\hline & & SI empleo-NO crianza & .45 & .878 & 78 \\
\hline & & NO empleo-SI crianza & .34 & .781 & 38 \\
\hline & & NO empleo-NO crianza & .58 & 1.091 & 33 \\
\hline & & Total & .40 & .848 & 227 \\
\hline & \multirow{5}{*}{36 a 49} & SI empleo-SI crianza & .26 & .714 & 265 \\
\hline & & SI empleo-NO crianza & .48 & .969 & 103 \\
\hline & & NO empleo-SI crianza & .37 & .887 & 106 \\
\hline & & NO empleo-NO crianza & .63 & 1.001 & 43 \\
\hline & & Total & .35 & .838 & 517 \\
\hline & \multirow{5}{*}{50 o más } & SI empleo-SI crianza & .30 & .777 & 37 \\
\hline & & SI empleo-NO crianza & .24 & .712 & 107 \\
\hline & & NO empleo-SI crianza & .26 & .734 & 42 \\
\hline & & NO empleo-NO crianza & .31 & .810 & 124 \\
\hline & & Total & .28 & .760 & 310 \\
\hline & \multirow{5}{*}{ Total } & SI empleo-SI crianza & .27 & .721 & 380 \\
\hline & & SI empleo-NO crianza & .38 & .859 & 288 \\
\hline & & NO empleo-SI crianza & .34 & .831 & 186 \\
\hline & & NO empleo-NO crianza & .43 & .910 & 200 \\
\hline & & Total & .34 & .818 & 1054 \\
\hline
\end{tabular}

\section{Discusión}

Los resultados obtenidos ponen de manifiesto que la edad y el tipo de ocupación relacionada con el empleo y la crianza influyen en las barreras para la práctica de AFD de las mujeres adultas en España.

El empleo y la crianza influyeron en las 3 barreras de la D3 Ocupación empleo y tiempo. La falta de tiempo se comprobó como barrera muy importante para la práctica de AFD de las mujeres adultas (Amin et al., 2011;
Bautista et al., 2011; Cerin et al., 2010; De Bourdeaudhuij \& Sallis, 2002; European Commission, 2018; Louw et al., 2012; Sebastião et al., 2011; Seefeldt, et al., 2002; Sørensen \& Gill, 2008). Los resultados obtenidos evidencian que las mujeres que concilian empleo y crianza, independientemente de su edad, presentaron en mayor medida la barrera incapacidad de generar tiempo para sí mismas, así como incompatibilidad del empleo y los horarios para poder realizar práctica físico-deportiva de manera regular. En esta línea, en la investigación llevada a cabo por Espada et al. (2019) las mujeres que practican AFD de entre 30 y 59 años eligen principalmente practicar de manera autónoma, podría deducirse que esta práctica libre y autónoma les permite compatibilizar con el empleo y la crianza.

Por esta razón, parece recomendable realizar estudios cualitativos para ampliar el conocimiento de la relación de esta incapacidad con la ética del cuidado familiar, como apunta el estudio de Gonzáles (2004).

La edad influyó en las 2 barreras de la D2 Ocupación crianza y hogar, relacionadas con los roles de cuidado de género, tradicionalmente atribuidos a las mujeres.

En relación a las tareas del hogar como barrera, además de la edad influyeron la crianza y el empleo. Se observaron diferencias en función de la edad en el grupo de mujeres empleadas que no son madres. Esta barrera se presentó en mayor medida en las mujeres más mayores, de 50 años y más. Esta revelación pudiera sugerir cambios en los patrones culturales en las adultas que no son madres ya que, para el mismo nivel de ocupación, las más mayores mencionaban esta barrera en mayor medida, es decir, una mayor prevalencia de este rol de género entre las adultas de mediana edad y, por el contrario, menor en las adultas y las jóvenes adultas. Pero también pudiera ser que las de menor edad no realicen estas tareas porque cuentan con ayuda externa a la familia remunerada, es decir, porque estas tareas de cuidado del hogar se hayan delegado a empleadas de hogar, dado que estas tareas las realizan principalmente mujeres.

En relación a la crianza como barrera la edad influyó, pero no el empleo, dado que esta barrera se presentó en mayor medida cuanto menor era la edad de las mujeres, independientemente de si eran madres empleadas o no. No obstante, son las jóvenes adultas el grupo de edad que presentó mayores puntuaciones, coincidiendo este último resultado con los obtenidos por el estudio de Moscoso et al. (2008). Se corrobora así los resultados de la investigación llevada a cabo por Isorna, Felpeto, Alonso, Gómez y Rial (2019) en la que anali- 
zan los motivos por los cuales las piragüistas han abandonado la práctica deportiva y concluyen que el caso de la maternidad es una de las situaciones donde ellas describen como un ejemplo de clara desigualdad: abandono por parte de las entidades deportivas cuando se tiene un hijo o cuando no se tienen los resultados deportivos esperados post maternidad.

Los resultados obtenidos en la presente investigación amplían y complementan el conocimiento proporcionado sobre la influencia del triple rol femenino en la brecha deportiva de género y edad (Moscoso y Rodríguez, 2020) y otros estudios sobre las barreras para la práctica de AFD de las mujeres adultas (Amin et al.,2011; Cerin et al., 2010; Martínez del Castillo et al., 2005; Moscoso et al., 2008; Sorensen \& Gill, 2008).

En especial, evidencia que las mujeres que concilian empleo y crianza representan el grupo con mayor incapacidad de generar tiempo para sí mismas, los horarios incompatibles y el empleo como barreras. También se desprende que la crianza es una barrera tan importante para las madres empleadas como para las desempleadas, así como su mayor prevalencia entre las madres más jóvenes. Por último, las tareas del hogar como barrera para la práctica de AFD influyen más en las mujeres que no son madres de mediana edad y menos en las adultas y jóvenes adultas que tampoco son madres.

Basándose en la evidencia de las dificultades de las madres para practicar AFD, diferentes estudios y herramientas recomiendan adaptar la oferta de AFD proporcionando cuidados, ludoteca mejor que guardería por ser el juego un precursor del deporte, así como coordinar la diferente oferta de actividades infantiles y para adultas en el mismo tiempo y espacio (Consejo Superior de Deportes-Federación Española de Municipios y Provincias, 2011; Council of Europe, 2019; Martín et al.; 2014).Así mismo, el estudio referencial From Brighton to Helsinki. Women and Sport Progress Report 1994-2014 (Fasting, Sand, Pike \& Matthews, 2014) concretaba que el desarrollo de provisión de cuidados infantiles para las personas involucradas en el deporte en diferentes roles y áreas, es una de las cuatro acciones prioritarias para mejorar la participación de las mujeres en el deporte.

Otras innovaciones propuestas fueron la oferta de programas de práctica conjunta infancia y personas adultas, que fue la más solicitada por las mujeres y hombres adultos de la demanda latente (no practicantes que desean practicar) del estudio de Martín et al. (2014). Cabe mencionar que esta demanda latente estuvo integrada mayoritariamente por madres empleadas con hijos menores de 8 años. Con especial atención a las madres empleadas, se añadían a estas propuestas, la oferta de programas de ejercicio físico en el trabajo, en el propio centro o mediante convenio con instalaciones y organizaciones deportivas próximas.

A las dificultades habituales que tenían las mujeres adultas para conciliar práctica de AFD con crianza y empleo, actualmente hay que añadir el impacto de la pandemia COVID-19. Los déficits de corresponsabilidad comunitarios y familiares han centralizado en ellas las tareas de cuidado (Odunaiya, Aderibigbe \& Oguntibeju, 2011). A esto hay que sumar la sobrecarga del feminizado empleo sanitario y de servicios esenciales, su mayor precariedad laboral y riesgo de pobreza, así como el incremento de la violencia de género; por ello, incorporar la perspectiva de género es esencial en la respuesta al COVID-19 (Instituto de la Mujer y para la Igualdad de oportunidades, 2020a). En relación a los cambios en los hábitos de práctica de AFD de las mujeres adultas en España consecuencia del COVID-19, Martín (2020) recogiendo la evidencia pre y post disponible, así como las propuestas de organizaciones de referencia, como el IWG responds to COVID 19 with global 'Call to Action', indicaba que las mujeres disminuyeron la intensidad y cantidad de práctica de AFD.

Como principales limitaciones del presente trabajo cabe destacar el marcado corte transversal o seccional de la recogida de datos. Como futura línea de investigación sería interesante seguir midiendo las barreras que perciben las mujeres para la practica de AFD y de esta manera comprobar si permanecen estables en el tiempo. Por otra parte, sería interesante conocer más en profundidad el entorno familiar para conocer cuáles son las barreras que limitan la practica de actividad física en el núcleo familiar.

\section{Conclusiones}

La edad y el tipo de ocupación relacionada con el empleo y la crianza influyen en las barreras para la práctica de AFD de las mujeres adultas en España relacionadas con las ocupaciones.

Las mujeres que concilian empleo y maternidad o crianza, independientemente de su edad, presentaron en mayor medida las barreras incapacidad de generar tiempo para sí mismas, el horario del empleo no les permite compatibilizar con los horarios para poder realizar práctica físico-deportiva de manera regular.

En madres adultas, empleadas o no, la crianza es una barrera que se presenta en mayor medida cuanto menor es la edad de las mujeres. 
En las mujeres que no son madres, la edad influyó en el cuidado del hogar como barrera, teniendo mayor prevalencia entre las mujeres de mediana edad.

Sería recomendable que estas barreras se tuvieran en cuenta en la promoción y adaptación de una oferta deportiva al conjunto de las mujeres adultas conciliadora con su crianza y empleo, con especial atención a las jóvenes madres empleadas o no.

\section{Nota}

La investigación aquí presentada ha formado parte del proyecto Investigación social de las necesidades, barreras e innovaciones en la oferta deportiva a las mujeres adultas en España, financiado por el Plan Nacional I+D+i del Ministerio de Ciencia e Innovación. DEP2010-19801

\section{Referencias}

Abbasi, I.N. (2014). Socio-cultural Barriers to Attaining Recommended Levels of Physical Activity among Females: A Review of Literature. Quest, 66(4), 448-467. Doi: 10.1080/00336297.2014.955118

Amin, T.T.; Suleman, W.; Ali, A.; Gamal, A., \& Al Wehedy, A. (2011). Pattern, Prevalence, and Perceived Personal Barriers Toward Physical Activity Among Adult Saudis in Al-Hassa, KSA. Journal of Physical Activity and Health, 8, 775-784. Doi: 10.1123/jpah.8.6.775.

Bauman, A. E., Reis, R.S., Sallis, J.F., Wells, J.C., Loos, R.J.F. \& Martin, B.W. 2012). Correlates of physical activity: why are some people physically active and others not? Lancet Journal, 380(9838), 258-271. Doi :https://doi.org/10.1016/S0140-6736(12)60735-1

Bautista, L.; Reininger, B.; Gay, J.L.; Barroso, C.S., \& McCormick, J.B. (2011). Perceived Barriers to Exercise in Hispanic Adults by Level of Activity. Journal of Physical Activity and Health, 8(7), 916-925. Doi: 10.1123/ jpah.8.7.916

Bronfenbrenner, U. (1993). The ecology of cognitive development: research models and fugitive findings. In: R.H.Wozniak and K.W. Fisher, eds. Development in context: activity and thinking in specific environments. Hillsdale, NJ: Erlbaum, 3-24.

Castañeda-Babarro, A.; Arbillaga-Etxarri, A.; GutiérrezSantamaría, B., \& Coca, A. (2020). Cambio de actividad física durante el encierro por COVID-19. International Journal of Environmental Research and Public Health ,17(18): 6878. doi: 10.3390 / ijerph17186878

Cerin. E.; Leslie, E.; Sugiyama, T., \& Owen, N. (2010). Perceived barriers to leisure-time physical activity in adults: an ecological perspective. Journal of Physical Activity and Health, 7, 451-459. Doi: 10.1123/jpah.7.4.451

Consejo Superior de Deportes-Federación Española de Municipios y Provincias (2011). Guía para la incorporación de la perspectiva de género a la gestión deportiva local. Madrid: CSD-FEMP. Disponible en https:// sede.educacion.gob.es

Council of Europe (2019). TOOLKIT How to make an impact on gender equiality in sport:All youneed to know. Bruselas: Council of Europe. Disponible en https://pjp-eu.coe.int/en/ web/gender-equality-in-sport/toolkit

De Bourdeaudhuij, I., \& Sallis, J. (2002). Relative Contribution of PsychosocialVariables to the Explanation of Physical Activity in Three Population-Based Adult Samples. Preventive Medicine, 34, 279-288. Doi: 10.1006/ pmed.2001.0979

Espada, M., Martín, M., Jiménez, V., Moscoso, D., JiménezBeatty, E. \& Rivero, A. (2018). Leisure sports habits in Spanish adults: gender and social differences. OBETS. Revista de Ciencias Sociales, 13(2): 495-513. doi: 10.14198/ OBETS2018.13.2.02

Espada, M., Martín, M., Jiménez, V., del Hierro, D., Santacruz, J.A. \& Jiménez-Beatty, E. (2019). Organizational aspects of adults' sports habits in Spain: gender and social differences, Motricidade, 15(1), 24-32. http://dx.doi.org/10.6063/motricidade.13997

Espada, M. \& Galán, S.(2017). Evaluación de las barreras para la práctica de actividad física y deportiva en los adolescentes españoles, Revista de Salud Pública, 19(6), 739743. DOI: https://doi.org/10.15446/ rsap.V19n6.66078

European Commission (2018). Special Eurobarometer 472. Report Sport and physical activity. European Union. Disponible en https://ec.europa.eu/commfrontoffice/ publicopinion/index.cfm

Fasting, K.; Sand, T.S.; Pike, E. \& Matthews, J. (2014). From Brighton to Helsinki.Women and Sport Progress Report 19942014. Valo: International Working Group Women and Sport-Finish Sport Confederation.

García-Tascón, M., Mendaña-Cuervo, C., Sahelices-Pinto, C. \& Magaz-González, A. (2021). Repercusión en la calidad de vida, salud y práctica de actividad física del confinamiento por Covid-19 en España. Retos, 42, 684-695

Gonzales, A. (2004). Mi familia viene primero (My family comes first): Physical Activity Issues in Older Mexican American Women. Southern Online Journal of Nursing Research, 4,5,2-21

Instituto Nacional de Estadística (2020). Demografia y población. https://www.ine.es/dyngs/INEbase/es/ categoria.htm?c=Estadistica_P\&cid $=1254735572981$ 
Instituto de la Mujer y para la Igualdad de oportunidades (2020a). La perspectiva de género, esencial en la respuesta a la COVID-19. Madrid: Ministerio de Igualdad - Instituto de la Mujer y para la Igualdad de oportunidades. Disponible en https: / / www.inmujer.gob.es

Instituto de la Mujer y para la Igualdad de oportunidades (2020b). Mujeres en Cifras-Conciliación-Usos del tiempo. Disponible en https://www.inmujer.gob.es

International Working Group on Women and Sport (2020).

ALERT: IWG responds to COVID-19 with global 'Call to Action'. Disponible en https:// iwgwomenandsport.org/es/iwg-responds-to-covid-19pandemic-with-global-call-to-action/

Isorna, M., Felpeto, M., Alonso,D., Gómez, P. \& Rial, A. (2019). Mujer y piragua: estudio de las variables moduladoras del abandono deportivo de las mujeres piragüistas en modalidades olímpicas, Retos, 35, 320-325

García-Ferrando, M. \& Llopis, R. (2011). Ideal democrático y bienestar personal. Encuesta sobre hábitos deportivos en España 2010. Madrid: Consejo Superior de Deportes -Centro de Investigaciones Sociológicas. Disponible en https: / / libreria.cis.es

García-Tascón, M.; Sahelices-Pinto, C.; Mendaña-Cuervo, C., \& Magaz-González, A.M. (2020). El impacto del encierro por COVID-19 en los hábitos de práctica de AF según género (hombre / mujer): caso español. Int J Environ Res Salud Pública 17(19): 6961. Doi: 10.3390 / ijerph17196961

Lefaivre, Liane. (2015). Ground-up city: Play as a design tool. Rotterdam: 010 Publishers.

López Martínez, J.J.; Rodríguez-Roiz, J., \& Salcedo Cánovas, C (2020). Lesiones musculoesqueléticas secundarias al ejercicio durante el encierro por la pandemia COVID19. Med Clin 155 (5): 221-222. Doi: 10.1016 / j.medcle.2020.05.013

Louw, A.J.; Van Biljon, A., \& Mugandani, S.C. (2012). Exercise motivation and barriers among men and women of different age groups. African Journal for Physical, Health Education, Recreation and Dance, 18,4(1), 759-768.

Martín, M. (2020). COVID-19 y práctica de actividad física y deporte entre mujeres adultas españolas: Contexto y recomendaciones. Sociología del Deporte (SD) , 1 (2), 71 76.DOI: http://doi.org/10.46661/socioldeporte.5429

Martín, M. (dir.); Martínez del Castillo, J.; Fasting, K.; Barriopedro, M.; Jiménez-Beatty, J.E.; Rivero, A... . (2014). Proyecto $I+D+i$ Necesidades, barreras e innovaciones en la oferta deportiva a las mujeres adultas en España: Informe para profesionales y organizaciones deportivas. Madrid: Grupo de Investigación psicosocial en el deporte - Universidad Politécnica de Madrid. Disponible en http:// investigacionsocialdeporte.com
Martínez del Castillo, J.; Vázquez, B.; Graupera, J.L.; Jiménez-Beatty, J.E.; Alfaro, E.; Hernández, M. \& Avelino, D. (2005). Estudio sobre la actividad fisica y deportiva de las mujeres del municipio de Madrid: Hábitos, demandas y barreras. Madrid: Ayuntamiento de Madrid

McGuire, A.; Seib, C., \& Anderson, D. (2016). Factors predicting barriers to exercise in midlife Australian women. Maturitas, 87, 61-66. Doi: 10.1016/ j.maturitas.2016.02.010

Ministerio de Cultura y Deporte (2020). Anuario de estadísticas deportivas 2020.Madrid: Secretaría General TécnicaMinisterio de Cultura y Deporte. Disponible en https: / /www.culturaydeporte.gob.es

Moscoso, D., Moyano E., Biedma, L., Fernández-Ballesteros, R., Martín, M., Ramos, C., Rodríguez-Morcillo, L. \& Serrano, R. (2008). Deporte, salud y calidad de vida. Colección Estudios Sociales no 26. Barcelona: Fundación La Caixa.

Moscoso, D. \& Rodríguez, A. (2020). El sesgo de la participación deportiva en España: argumentos y análisis alternativos. En Diálogos sobre el deporte (1975-2020), 15 - 32. Barcelona:

Odunaiya, N.A., Aderibigbe, A.A. \& Oguntibeju, O.O. (2011). Physical exercise: knowledge, attitudes and habits of literate women in Westem Nigeria. African Journal for Physical, Health Education, Recreation and Dance, 17(4:2): 789-803.

Piedra, J. (2020). Redes sociales en tiempos del COVID19: el caso de la actividad física. Sociología del Deporte 1(1): 15-19. Doi: http://doi.org/10.46661/ socioldeporte.4998

Sánchez-García, R. (2020). La reutilización lúdica del espacio urbano en tiempos de la COVID-19. Sociología del Deporte 1(1): 25-28. Doi: http://doi.org/10.46661/ socioldeporte. 4999 .

Sebastião, C.; Pinto, D.; Santos, L.; Marques, A., \& Carreiro da Costa, F. (2011). Prevalence of barriers for physical activity in adults according to gender an socioeconomic status. British Journal of Sports Medicine, 45:e5. Doi:10.1136/A18bjsports-2011-090606

Seefeldt, V.; Malina, R.M., \& Clark, M. (2002). Factors affecting levels of physical activity in adults. Sports Medicine, 32(3), 143-168. Doi: 10.2165/00007256-20023203000001

Sørensen, M., \& Gill, D. (2008). Perceived barriers to physical activity across Norwegian adult age groups, gender and stages of change. Scandinavian Journal of Medicine \& Science in Sports, 18(5), 651-663. Doi: 10.1111/ j.1600-0838.2007.00686.x 